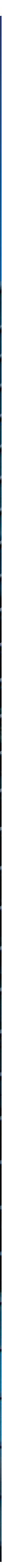




\section{User Psychology Lab, University of Jyväskylä}

As told by Rebekah Rousi, Tuomo Kujala, Johanna Silvennoinen, Laura Mononen, Naomi Woods, Hilkka Grahn, and Pila Perälä

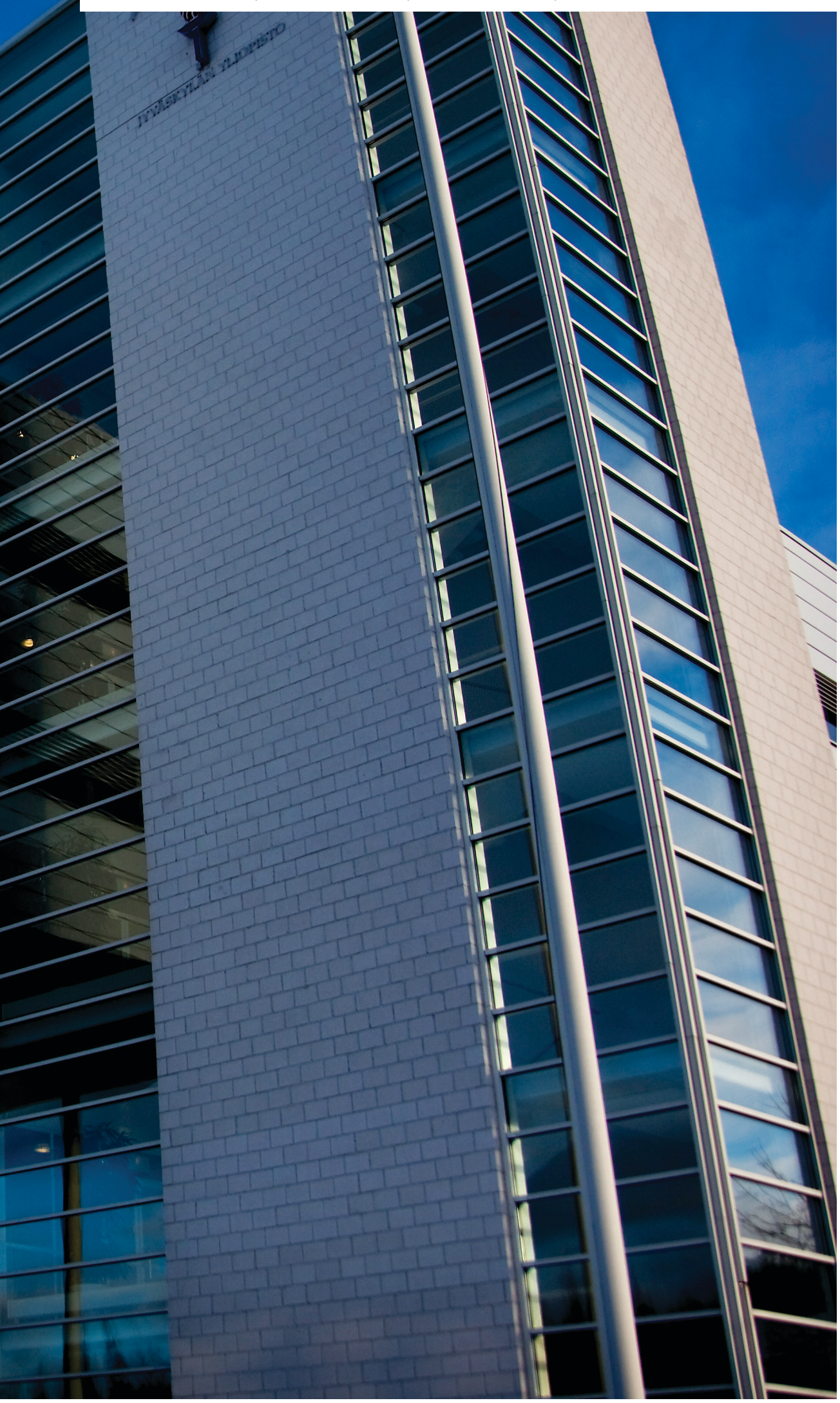

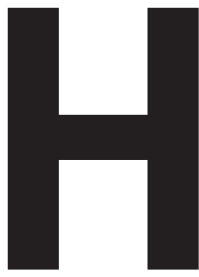

ow do you describe your lab to visitors? At the User Psychology Lab, University of Jyväskylä, Finland, our main focus is on humantechnology interactions.

Visitors can expect to see people interacting with any number of weird and wonderful designs, from in-vehicle displays to glassware and frying pans. Although this scope may seem daunting, the link between all the studies is the person (human) - their cognitive capacity; their embodied cognition (from driver performance to affective interactions); their cognitive-affective processes; and the technological designs established to (ideally) improve the quality of everyday lived experience. One of the underlying frameworks of the User Psychology Lab is Life-Based Design. Life-Based Design rejects ideas of the typical user, instead focusing on cohorts such as age to define the change in interactions and experiences in various technology usage situations.

The research done in the lab emphasizes a deep multidisciplinary understanding of human beings as the basis of interaction design. This involves various fields that study the human mind, thought, and behavior, such as cognitive science, psychology, and sociology. Relevant topics of research include (but are not limited to) human factors and ergonomics, user psychology, usability, user experience (UX), human-computer interaction (HCI), and at the most general level, what we like to call human-technology interaction (HTI).

The laboratory is well equipped for conducting high-quality experimental basic research with eye-tracking measures, driving simulators, EEG, and basic behavioral research tools. Applied research is often conducted with Finnish and international industrial collaborators. The coordination of basic and applied research affects both the understanding of the life and behaviors of technology users and the development of actual technologies based on that understanding.

How many people are in the lab, and what is the mix of backgrounds and roles? Our small Cognitive Science team (fewer than 10 people) is highly interdisciplinary, with researchers from information systems, philosophy, 

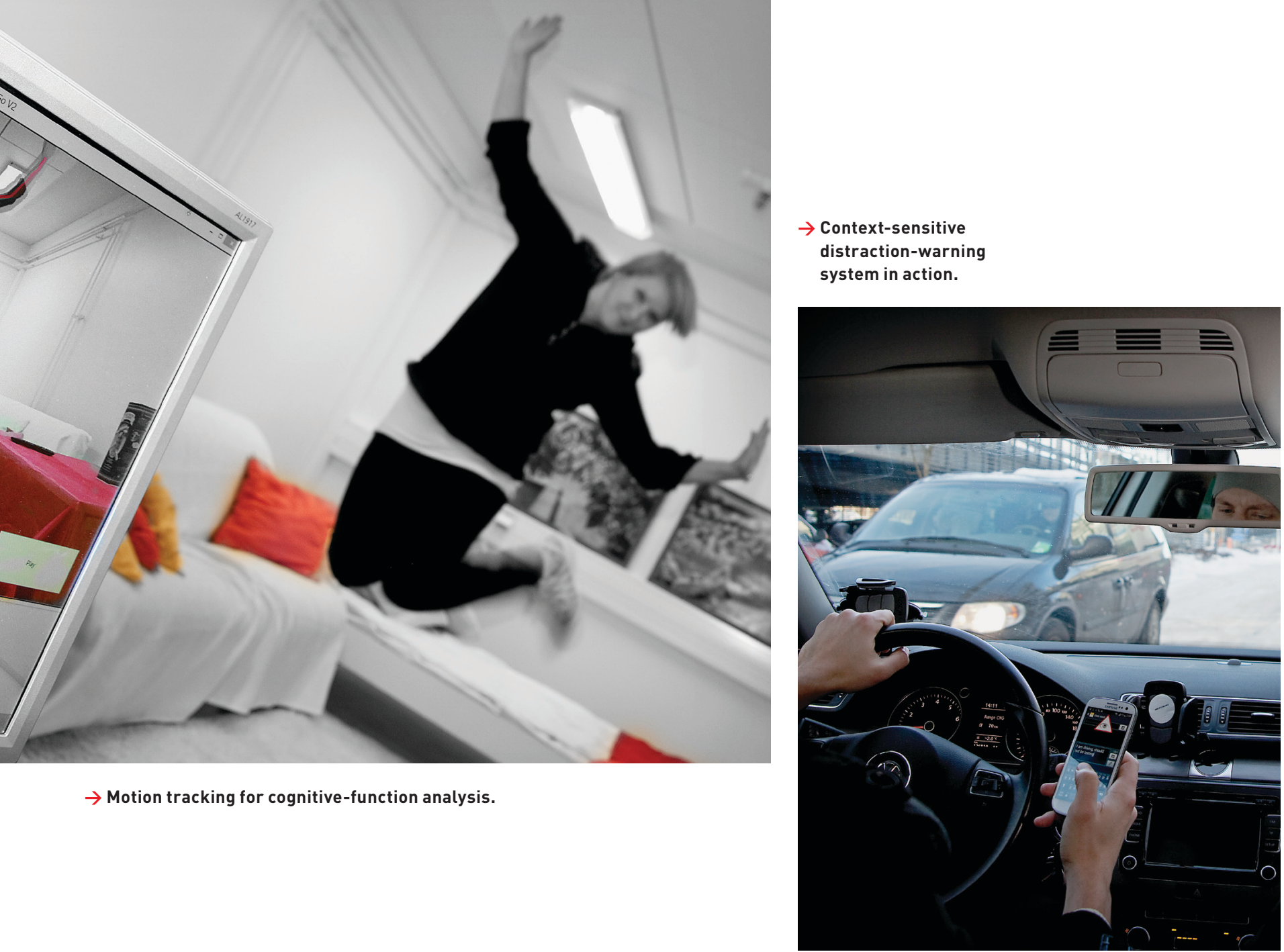

The lab itself can be found in various locations of the Agora building in Mattilanniemi, with a domestic-life simulator and a driving simulator in the basement, and a soundproof, interrogation-style two-way mirror setup for reaction time and EEG measurements on the third floor. However, we pride ourselves on our flexibility and on our ability to conduct studies and simulations anywhere, at any time, with a DIY attitude.

\section{How would you describe how} people interact in your lab? We are a progressive bunch of people, in teamwork and discussions, as well as with students. We have moved from an open door policy to a no door policy. We know all the cognitive science students by name and are fairly good at remembering many others within the Faculty of Information Technology and across the university. We hassle our students and staff with regular announcements to recruit participants for our often weird-sounding experiments. During the past few years we have studied, for example, how well you can drive a car without vision, what vacuum cleaning looks like through motion-sensing cameras, and the experiences people have while touching different materials confined in a box.

\section{What is one feature of your lab that} you could not do without? What we can't live without are our wonderful support staff - the research assistants, who are underpaid and overworked, yet still smiling all the time. We also could not have succeeded in building this environment without our open-minded professor Pertti Saariluoma, who has pulled together a hardworking, forward-thinking, dynamic team, and who never ceases to inspire in terms of new ideas and connections.

What is the one thing you see as most important about the work you do there? Even if we are studying people's interactions with technology, one could say that instead of technology, we are more interested in better understanding the human mind and behavior in interaction with technology. Whereas technological trends move and change rapidly, the human in HTI (or HCI) changes more slowly. We believe that better understanding of the $\mathrm{H}$ in HTI can lead to better HTI and more intelligent technology, where the intelligence is measured by the quality of communication, interaction, and cooperation with people.

https://www.jyu.fi/it/laitokset/cs/en/ research/hti/hti 